Diabetologia (1994) 37: 905-910

Diabetologia

(C) Springer-Verlag 1994

\title{
Abnormal increases in urinary albumin excretion during pregnancy in IDDM women with pre-existing microalbuminuria
}

\author{
G. Biesenbach ${ }^{1}$, J.Zazgornik ${ }^{1}$, H.Stöger ${ }^{2}$, P. Grafinger ${ }^{1}$, R.Hubmann ${ }^{1}$, W. Kaiser ${ }^{1}$, O.Janko ${ }^{1}$, U. Stuby ${ }^{1}$ \\ ${ }^{1}$ II Department of Medicine, Nephrology Section, General Hospital, Linz, Austria \\ ${ }^{2}$ Department of Obstetrics and Gynecology, General Hospital, Linz, Austria
}

Summary We compared urinary albumin excretion during and after pregnancy in 30 insulin-dependent diabetic (IDDM) women with normoalbuminuria and in $12 \mathrm{IDDM}$ women with microalbuminuria $\left(>15 \mu \mathrm{g} \cdot \mathrm{min}^{-1}\right)$ prior to conception. There was a 6.7 fold increase in the urinary albumin excretion up until the third trimester in the women with pre-existing microalbuminuria, compared with a 3.8 -fold increase in the normoalbuminuric women. In both groups of patients the urinary albumin excretion reached a peak during the third trimester with $492 \pm 404 \mu \mathrm{g} \cdot \mathrm{min}^{-1}$ in the microalbuminuric women vs $43 \pm 36 \mu \mathrm{g} \cdot \mathrm{min}^{-1}$ in the normoalbuminuric women $(p<0.0005)$. Two women from each of the groups developed eclampsia with diastolic blood pressure over $90 \mathrm{~mm} \mathrm{Hg}$, mild or moderate oedema and macroproteinuria. Four of the pregnant women with pre-existing microalbuminuria showed a transient nephrotic syndrome $(33.3 \%)$ with protein excretion over $3 \mathrm{~g}$ in 24 -h urine samples during the third trimester. In contrast, this was not observed in any of the normoalbuminuric women $(p<0.05)$.
Within 12 weeks after delivery the urinary albumin excretion rates dropped to the pre-conception values in both patient groups. Renal function remained normal during pregnancy in both of the groups, with a physiological increase in creatinine clearance up until the third trimester $(26 \%$ increase in the normoalbuminuric women vs $22 \%$ in the microalbuminuric women). In conclusion, the effect of pregnancy on the urinary albumin excretion in diabetic women with preexisting microalbuminuria is an exaggeration of the increase of albuminuria in diabetic women with normoalbuminuria; normalization occurs within 12 weeks after delivery in all cases. This enhancement of the albumin excretion in the microalbuminuric women cannot be explained by a larger increase in the hyperfiltration during pregnancy. [Diabetologia (1994) 37: 905-910]

Key words Urinary albumin excretion, microalbuminuria, macroproteinuria, diabetic pregnancy, perinatal outcome.
A subclinical increase in urinary albumin excretion, socalled microalbuminuria (albumin excretion rate $>15 \mu \mathrm{g} \cdot \mathrm{min}^{-1}$ ), is the main manifestation of incipient diabetic nephropathy and a predictor of the later development of clinical diabetic nephropathy $[1,2]$. Glo-

Received: 21 December 1993

and in revised form: 29 March 1994

Corresponding author: Dr. G.Biesenbach, II Department of Medicine, General Hospital, Krankenhausstraße 9, A-4020 Linz, Austria

Abbreviations: IDDM, Insulin-dependent diabetes mellitus; NIDDM, non-insulin-dependent diabetes mellitus. merular hyperfiltration is a feature of IDDM and it can be assumed that the augmented filtration rate or some haemodynamic determinants of that increased rate, such as an elevated capillary pressure, damage the diabetic glomerulus directly [3]. A transient increase in the glomerular filtration rate can also be seen in healthy pregnancy [4], and this physiological hyperfiltration may be accompanied by a slight increase in the protein excretion up until the third trimester [5]. In diabetic women with normoalbuminuria prior to conception the effect of pregnancy on the urinary albumin excretion is obviously an exaggeration of the normal pattern; the levels return to the normal range after delivery [6]. But there are no data in the literature concerning the 
Table 1. Clinical and laboratory data prior to conception in diabetic women with normoalbuminuria and microalbuminuria

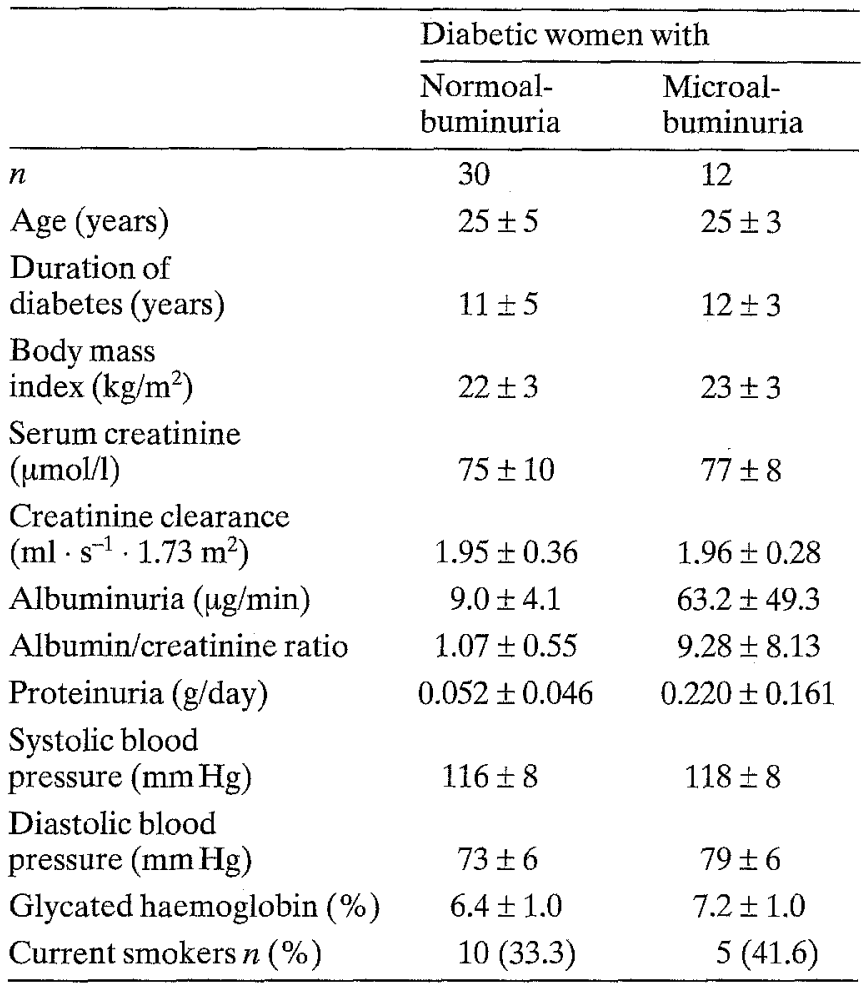

Values are shown as mean \pm SD, prevalences are presented as number (\%)

influence of pregnancy on urinary albumin excretion in diabetic women with pre-existing microalbuminuria.

We compared the urinary albumin excretion rates and the creatinine clearances before, during and after pregnancy in 30 IDDM women with normal albumin excretion prior to conception and in 12 IDDM women with pre-existing microalbuminuria. The aim of this prospective study was to evaluate the effect of pregnancy on urinary albumin excretion and renal function in diabetic women who have elevated albumin excretion even before pregnancy.

\section{Subjects and methods}

The urinary albumin excretion and renal function during and after pregnancy were compared in 30 IDDM women with preexisting normoalbuminuria and in 12 IDDM women with microalbuminuria (urinary albumin excretion $>15$ and $<250 \mu \mathrm{g} \cdot \mathrm{min}^{-1}$ ). All of these women were under out-patient hospital care even prior to conception and became pregnant during the years 1985-1992. Entry into the study was at the first attendance in our out-patient care unit after 8-12 weeks gestation. Data for the patients were available for at least 6 months prior to conception, during pregnancy and 3 months after delivery. Diabetic women with pre-existing macroproteinuria ( $>0.5 \mathrm{~g}$ protein $24-\mathrm{h}$ urine samples) were excluded from the study. The 42 patients were aged 18-43 years, the duration of diabetes ranged from $2-24$ years. The clinical and laboratory data of the diabetic women with pre-existing normoalbuminuria as well as the data of those with microalbuminuria, mea-
Table 2. Data of delivery, fetomaternal complications and perinatal outcome in diabetic women with pre-existing normoalbuminuria and microalbuminuria

\begin{tabular}{|c|c|c|}
\hline & \multicolumn{2}{|c|}{ Diabetic women } \\
\hline & $\begin{array}{l}\text { Normoal- } \\
\text { buminuria }\end{array}$ & $\begin{array}{l}\text { Microal- } \\
\text { buminuria }\end{array}$ \\
\hline Vaginal delivery & $16(53.3 \%)$ & $4(33.3 \%)^{b}$ \\
\hline Caesarean section & $14(46.6 \%)$ & $8(66.6 \%)^{b}$ \\
\hline $\begin{array}{l}\text { Gestational week } \\
\text { of delivery }\end{array}$ & $38 \pm 2$ & $37 \pm 2^{b}$ \\
\hline $\begin{array}{l}\text { Premature } \\
\text { delivery }(<34 \text { week })\end{array}$ & $0(0 \%)$ & $1(8.3 \%)^{b}$ \\
\hline Birthweight (g) & $3277 \pm 690$ & $3083 \pm 707^{b}$ \\
\hline $\begin{array}{l}\text { Small for } \\
\text { gestational age }\end{array}$ & $0(0 \%)$ & $2(16.6 \%)^{b}$ \\
\hline $\begin{array}{l}\text { Large for } \\
\text { gestational age }\end{array}$ & $1(3.3 \%)$ & $1(8.3 \%)^{\mathrm{b}}$ \\
\hline Maternal complications & & \\
\hline Polyhydramnios & $0(0 \%)$ & $1(8.3 \%)^{b}$ \\
\hline Eclampsia & $2(6.6 \%)$ & $2(16.6 \%)^{b}$ \\
\hline Nephrotic syndrome & $0(0 \%)$ & $4(33.3 \%)^{\mathrm{a}}$ \\
\hline Stillbirths & $0(0 \%)$ & $1(8.3 \%)^{b}$ \\
\hline Perinatal complications & & \\
\hline $\begin{array}{l}\text { Respiratory distress } \\
\text { syndrome }\end{array}$ & $0(0 \%)$ & $0(0 \%)$ \\
\hline $\begin{array}{l}\text { Hypoglycaemia } \\
\text { (blood glucose }<2.2 \mathrm{mmol} / \mathrm{l} \text { ) }\end{array}$ & $6(20 \%)$ & $4(33.3 \%)^{b}$ \\
\hline $\begin{array}{l}\text { Hyperbilitubinaemia } \\
(>205 \mu \mathrm{mol} / \mathrm{l})\end{array}$ & $2(6.6 \%)$ & $2(16.6 \%)^{\mathrm{b}}$ \\
\hline Major congenital anomalies & $0(0 \%)$ & $1(8.3 \%)^{b}$ \\
\hline
\end{tabular}

Data are given as mean \pm SD, prevalences as number (\%). ${ }^{\mathrm{a}} p<0.05 ;{ }^{\mathrm{b}} \mathrm{NS}$

sured on two occasions up to 6 months before pregnancy, are shown in Table 1. Thirty-four of the women were in their first pregnancy, and eight in their second. The diagnosis of microalbuminuria was based on at least two consecutive laboratory tests for albumin and creatinine in urine with albumin excretion over $15 \mu \mathrm{g} \cdot \mathrm{min}^{-1}$ and albumin/creatinine ratio over $2(\mu \mathrm{g} / \mathrm{dl}$ per $\mu \mathrm{mol} / \mathrm{l})$ in the absence urinary tract infection. In the diabetic women with normoalbuminuria metabolic control was maintained during pregnancy by intensified insulin therapy with multiple insulin injections daily for 28 subjects and in two subjects with insulin pumps for continuous subcutaneous insulin infusion. In the microalbuminuric group metabolic control was achieved by intensified insulin therapy in eight subjects and with an external insulin pump in four subjects. None of the patients received antihypertensive therapy during pregnancy and none were taking drugs other than insulin. All of the women delivered at between 33 and 40 weeks of gestation; the mean gestational week of delivery was 38 in the normoalbuminuric women and 37 in the microalbuminuric group. One stillbirth occurred in the 40th week of gestation in a women with pre-existing microalbuminuria. The data concerning deliveries, fetomaternal complications and the perinatal outcome are summarized in Table 2. Blood samples and 24-h urine samples, which were taken in the morning, were obtained before pregnancy, at 2-week intervals throughout the pregnancy and 12 weeks after delivery. The 24 -h urine samples were collected by the patients during a 
Table 3. Urinary protein excretion and renal function as well as metabolic control and blood pressure before, during and after pregnancy in diabetic women with pre-existing normoalbuminuria

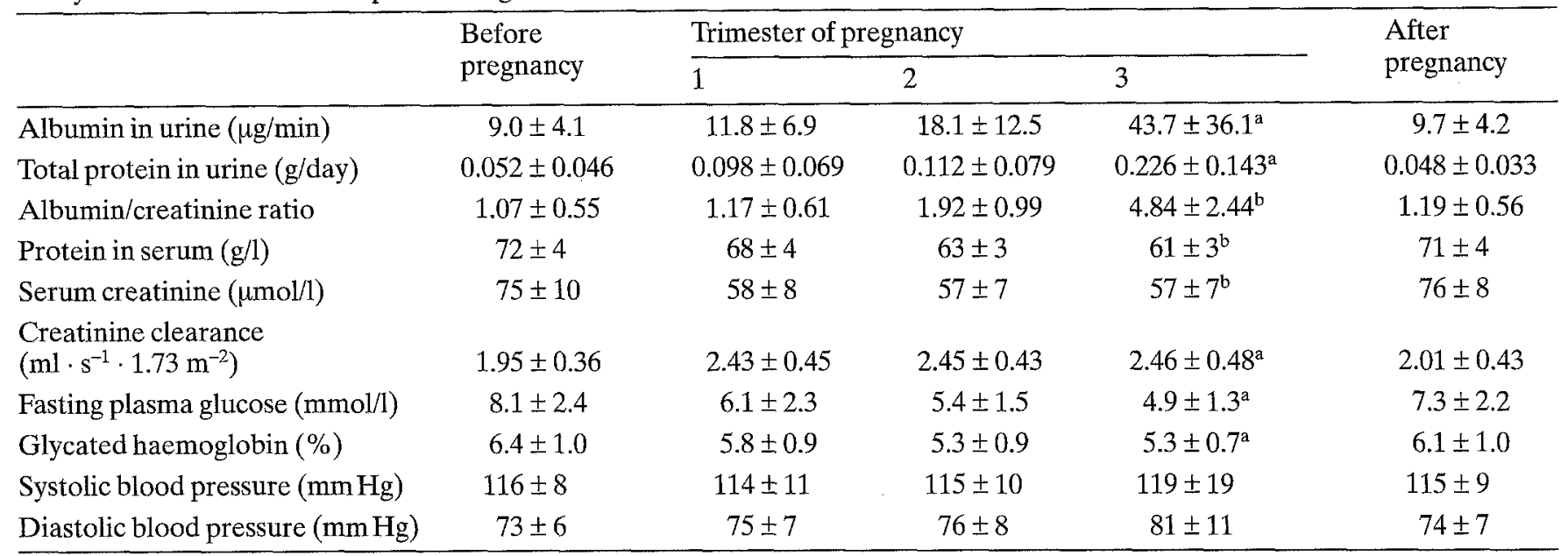

Values are given as mean $\pm \mathrm{SD}$

${ }^{\mathrm{a}} p<0.01,{ }^{\mathrm{b}} p<0.001$ significantly different between pre-pregnancy and third trimester data

resting day, according to instructions provided by one of the hospital nurses. In patients with suspected or proven urinary tract infection the estimation of the urinary albumin content was deferred until the culture was negative.

The following measurements were made at each visit to the clinic: fasting plasma glucose by the glucose oxidase method, creatinine in serum and urine as well as protein concentration in the serum using a multichannel autoanalyser (Hitachi, Boehringer Corporation, Vienna, Austria). Glycated haemoglobin was determined by microcolumn chromatography (Bio Rad Laboratories, Vienna, Austria; normal range: $4.2-5.9 \%$ ) and the total protein in the urine by the Biuret method. Urinary albumin excretion was measured by immunodiffusion (VLCPartigen Albumintest; Behring, Vienna, Austria) as described earlier $[7,8]$. The albumin/creatinine ratio was calculated. Body weight and blood pressure were recorded during the same visits, always in the morning; the blood pressure was measured after at least $15 \mathrm{~min}$ of rest in a supine position with a standard mercury sphygmomanometer. The creatinine clearance was calculated and corrected to $1.73 \mathrm{~m}^{2}$ body surface. Data are given from the visits before pregnancy, at each trimester of pregnancy (gestational week 12, 24 and on an average at week 38 in the normoalbuminuric women and at week 37 in the microalbuminuric women) and at 3 months postpartum.

\section{Statistical analysis}

Differences between groups were evaluated with the unpaired Student's $t$-test and comparisons within a group at different time-points were carried out with the paired Student's $t$-test. The statistical analysis of prevalences was performed with the chi-square test. All of the data are expressed as means \pm SD. A two-tailed significance level with $p$ less than 0.05 was applied.

\section{Results}

Changes in urinary protein excretion, renal function, metabolic control and blood pressure during pregnancy in the diabetic women with pre-existing nor- moalbuminuria are shown in Table 3. The mean albumin excretion in urine increased by $385 \%$ from $9.0 \pm 4.1 \mu \mathrm{g} \cdot \mathrm{min}^{-1}$ before conception to $43.7 \pm 36.1 \mu \mathrm{g} \cdot \mathrm{min}^{-1}$ at the third trimester pre-delivery $(p<0.01)$. The mean fractional albumin excretion (albumin/creatinine ratio) increased by $352 \%$ during the same period $(p<0.001)$. At 3 months postpartum the mean albumin excretion rates dropped to the values measured before conception. During pregnancy the creatinine clearance showed a transient physiological rise of $26 \%(p<0.01)$. The mean values of glycated haemoglobin and the systolic as well as diastolic blood pressure were normal throughout pregnancy.

For the diabetic women with pre-existing microalbuminuria the changes in urinary protein excretion, renal function, metabolic control and blood pressure during the same period are summarized in Table 4. The mean albumin excretion in urine increased by $678 \%$ from $63.2 \pm 49.3 \mu \mathrm{g} \cdot \mathrm{min}^{-1}$ prior to conception to $492.3 \pm 404.2 \mu \mathrm{g} \cdot \mathrm{min}^{-1}$ at the third trimester before delivery $(p<0.05)$. The albumin/creatinine ratios showed a similar trend with a rise of $601 \%$ during the same period $(p<0.05)$. At 3 months postpartum the urinary protein values were nearly the same as prior to conception. The creatinine clearances increased transiently by $22 \%$ during pregnancy $(p<0.05)$. The mean glycated haemoglobin was slightly elevated during the first trimester but normal in the second and third trimester. The average systolic and diastolic blood pressure increased slightly but not significantly during pregnancy.

The individual and mean urinary albumin excretion rates in pregnancy for both the diabetic women with pre-existing normoalbuminuria and the women with microalbuminuria prior to conception are shown in Figures 1 and 2. 
Table 4. Urinary protein excretion and renal function as well as metabolic control and blood pressure before, during and after pregnancy in diabetic women with pre-existing microalbuminuria

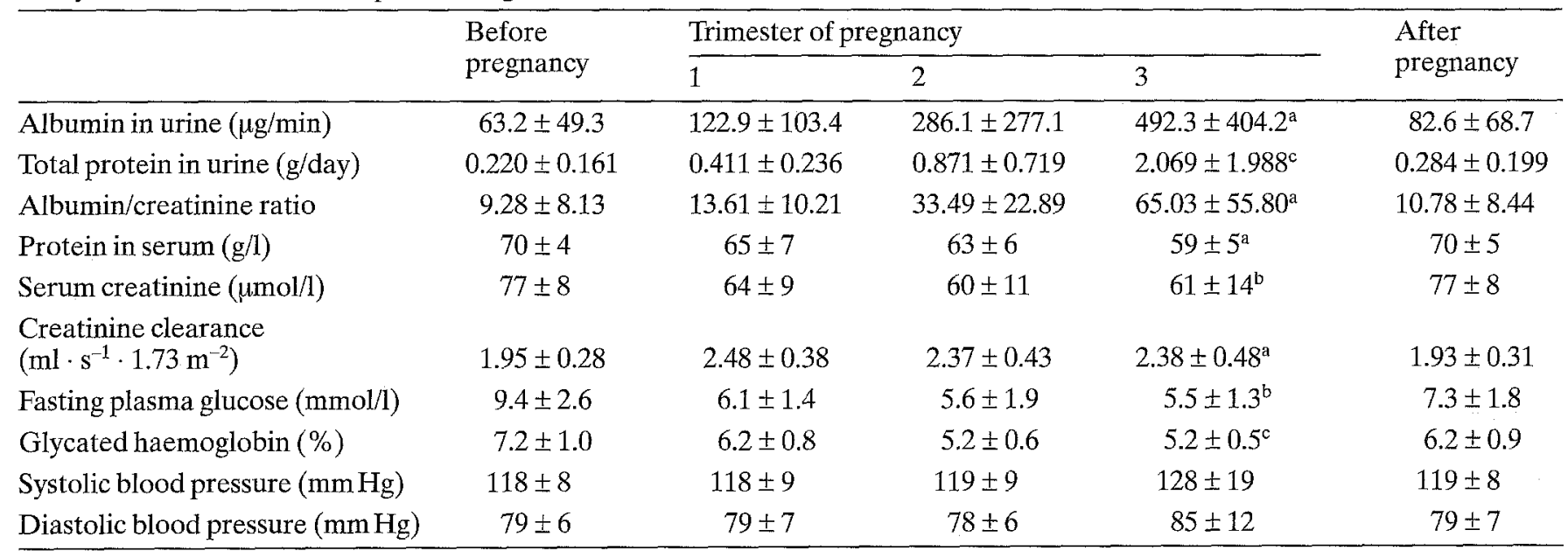

Values are shown as mean $\pm \mathrm{SD}$

${ }^{\mathrm{a}} p<0.05,{ }^{\mathrm{b}} p<0.01,{ }^{\mathrm{c}} p<0.001$ vs data before pregnancy

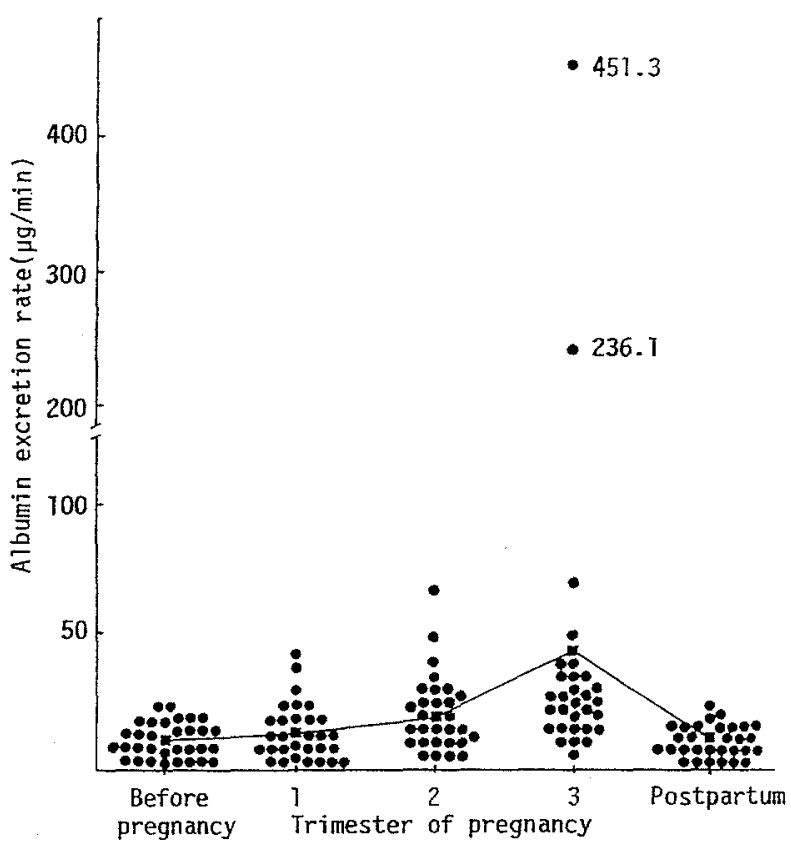

Fig. 1. Urinary albumin excretion before, during and after pregnancy in diabetic women with pre-existing normoalbuminuria. (-) median

The differences between the two groups of women were significant with respect to urinary albumin excretion at each visit before conception, during each trimester and at 3 months after delivery $(p<0.0005)$. The actual values of the albumin excretion rates increased from the time prior to conception until predelivery by $34.7 \pm 33.5 \mu \mathrm{g} \cdot \mathrm{min}^{-1}$ in the normoalbuminuric women and by $429.1 \pm 412.6 \mu \mathrm{g} \cdot \mathrm{min}^{-1}$ in the microalbuminuric women; this was an 11.3-fold greater increment in the latter group $(p<0.0005)$. Four of the microalbuminuric women showed an excessive increase in proteinuria with transient ne-

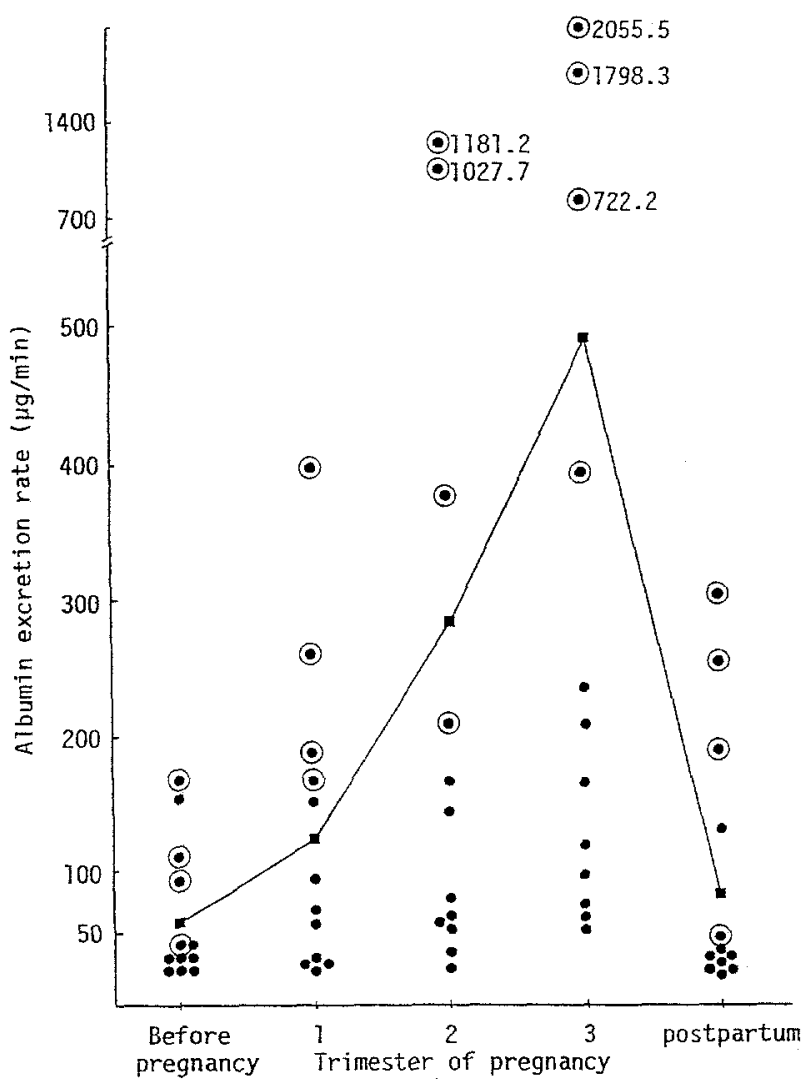

Fig. 2. Urinary albumin excretion before, during and after pregnancy in diabetic women with pre-existing microalbuminuria. (-) median. The four women with transient nephrotic syndrome $(\odot)$ showed also the highest albumin excretion rates during pregnancy

phrotic syndrome during the third trimester. But also in the other eight microalbuminuric women the actual values of the albumin excretion rates increased by $85.2 \pm 101.8 \mu \mathrm{g} \cdot \mathrm{min}^{-1}$ from the time before pregnancy until pre-delivery; this was a 2.4 -fold greater 
increment than in the normoalbuminuric women $(p<0.05)$.

The mean gestational week of delivery was nearly the same in both patient groups, but the Caesarian section rate was higher $(66.6 \%$ vs $46.6 \%$ ) in the microalbuminuric women (NS). The mean birth weight was $3277 \mathrm{~g}$ in the women with preexisting normoalbuminuria in comparison to $3083 \mathrm{~g}$ in the microalbuminuric group. Two babies of the women with microalbuminuria showed intrauterine growth retardation. Two women of each group developed eclampsia with elevated diastolic blood pressure (>90 $\mathrm{mm} \mathrm{Hg}$ ), oedema and macroproteinuria. A transient nephrotic syndrome could only be observed at the third trimester in four of the women with preexisting microalbuminuria, but in none of the normoalbuminuric women $(p<0.05)$.

In the women with transient nephrotic syndrome during pregnancy the mean urinary albumin excretion increased by $1084 \%$ from $103.4 \pm 51.3 \mu \mathrm{g} \cdot \mathrm{min}^{-1}$ prior to conception to $1220.1 \pm 678.4 \mu \mathrm{g} \cdot \mathrm{min}^{-1}$ pre-delivery. Total protein in urine increased by $1049 \%$ from $0.393 \pm 0.097 \mathrm{~g} /$ day to $4.515 \pm 1.515 \mathrm{~g} /$ day during the same period. Three of the four women showed higher initial albumin excretion rates of more than $70 \mu \mathrm{g} \cdot \mathrm{min}^{-1}$ (Fig. 2). The percent increase in the mean creatinine clearance was $10 \%$ during pregnancy (from $1.82 \pm 0.21$ to $2.01 \pm 0.31 \mathrm{ml} \cdot \mathrm{s}^{-1} \cdot 1.73 \mathrm{~m}^{-2}$ ). Three months after delivery the mean urinary protein excretion rates almost decreased to the values prior to conception. Mean glycated haemoglobin levels were slightly elevated at the first trimester but normal throughout the second and third trimester. The nephrotic women showed significantly higher systolic blood pressures at the third trimester compared to the the pre-pregnancy values $(133 \pm 9 \mathrm{~mm} \mathrm{Hg}$ vs $123 \pm 4 \mathrm{~mm} \mathrm{Hg}$ ).

\section{Discussion}

During normal pregnancy, glomerular filtration rate and effective renal plasma flow can rise $50-80 \%$ above non-pregnant levels, and a concomitant increase in the total proteinuria up to $0.3 \mathrm{~g} /$ day appears to be common [9]. Lopez-Espinoza et al. [10] and Gero et al. [11] also described a significant rise in the urinary albumin excretion in normal pregnant women, which peaked within 1 week before delivery; but the increase in albuminuria during pregnancy in healthy women is lower than in diabetic women. McCance et al. [6] found in IDDM women with normoalbuminuria at the time of conception a progressive increase in the urinary albumin excretion during the third trimester in comparison to a group of non-diabetic pregnant women.

In our diabetic women with microalbuminuria prior to conception the urinary albumin excretion increased proportionally to a significant 1.8 -fold greater extent during pregnancy when compared to the diabetic women with pre-existing normoalbuminuria. The albumin/creatinine ratios showed almost identical trends. The difference between the actual increase in the albumin excretion rates in both groups the patients with and without microalbuminuria - was highly significant $(p<0.0005)$. Four of the microalbuminuric women developed transient nephrotic syndrome during the third trimester; but also in the other eight microalbuminuric women without nephrotic syndrome the increase in the actual values of the albumin excretion rates during pregnancy was significantly higher than in the normoalbuminuric women $(p<0.05)$. Three of the four women with nephrotic syndrome showed higher initial albumin excretion rates of more than $70 \mu \mathrm{g} \cdot \mathrm{min}^{-1}$ in contrast to only one of the other microalbuminuric patients. Three months after delivery the protein excretion rates and the albumin/creatinine ratios were nearly the same as prior to conception in all cases. There was a similar physiological rise in the creatinine clearance during pregnancy in the patient groups with and without microalbuminuria ( 22 vs $26 \%$ ). Thus, the higher increment in albumin excretion in the pregnant women with pre-existing microalbuminuria cannot be explained by a higher increase in the glomerular filtration rate.

In the literature the development of the nephrotic syndrome has been usually described in pregnant diabetic women, but most of them had shown macroproteinuria even prior to conception [12-15]. Among our diabetic women four with preexisting microalbuminuria developed a transient nephrotic syndrome during the third trimester, but this occurred in none of the women with normoalbuminuria. Two patients developed eclampsia before delivery. The mean creatinine clearance of the four women with nephrotic syndrome increased by only $10 \%$ during pregnancy. In normal pregnancy the elevated glomerular filtration rate leads to an increased renal plasma flow but not to changes in the intraglomerular pressure [16]. In our diabetic women with nephrotic syndrome the systolic blood pressure was significantly higher in the third trimester; it can be assumed that an elevation of the glomerular pressure could be in part responsible for the greater increase in the proteinuria.

In conclusion, diabetic women with pre-existing microalbuminuria show a significantly higher increase in urinary albumin excretion during pregnancy than pregnant women with normoalbuminuria prior to conception. This exaggeration of the albumin excretion cannot be explained by greater increase in hyperfiltration during pregnancy. In all cases the elevated albumin excretion rates return to the values prior to conception. In addition, microalbuminuric diabetic women show a higher risk for the development of a transient nephrotic syndrome during the third trimester. 


\section{References}

1. Mogensen CE, Christensen CK (1984) Predicting diabetic nephropathy in insulin-dependent patients. New Engl J Med 311: 89-93

2. Viberti G, Jarret R, Mahmud U, Hill R, Argyropoulos K, Keen H (1982) Microalbuminuria as a predictor of clinical nephropathy in insulin-dependent diabetes mellitus. Lancet I: $1430-1432$

3. Hofstetter TH (1985) Diabetic nephropathy. New Engl J Med 312: 642-643

4. Davison JM (1987) Overview: kidney function in pregnant women. Am J Kidney Dis 9: 252-258

5. Friedberg V (1980) The kidney in normal pregnancy. Nieren- und Hochdruckkrankheiten 2: 53-59

6. McCance DR, Traub AI, Harley JMG, Hadden DR, Kennedy L (1989) Urinary albumin excretion in diabetic pregnancy. Diabetologia 32: 236-239

7. Mancini G, Carbonara AO, Heremans JF (1965) Immunochemical quantitation of antigens by single radial immunodiffusion. Immunochemistry 2: 235-239

8. Viberti GC, Wiseman M, Redmond S (1986) Microalbuminuria. Its history and potential for prevention of clinical nephropathy in diabetes mellitus. Diabetic Nephropathy $4: 79-82$

9. Davison JM (1989) Renal function during normal and abnormal pregnancy. Nieren- und Hochdruckkrankheiten 18: 311-319
10. Lopes-Espinoza I, Dhar H, Humphreys S, Redman WG (1986) Urinary albumin excretion in pregnancy. Br J Obstet Gynaecol 93: 176-181

11. Gero G, Davis FAM, Richardson MC, Dennis KJ, Rowe DJF (1987) Retinal-binding protein, albumin and total protein excretion pattern during normal pregnancy. J Obstet Gynecol 8: 104-108

12. Biesenbach G, Stöger H, Zazgornik J (1987) Changes in proteinuria and renal function in female type I diabetics during and after pregnancy dependent on the stages of preexisting diabetic nephropathy. Klin Wochenschr 65: 10481053

13. Kitzmiller JL, Brown ER, Philippe M et al. (1981) Diabetic nephropathy and perinatal outcome. Am J Obstet Gynecol 141: 741-751

14. Jovanovic R, Jovanovic J (1984) Obstetric management when normoglycemia is maintained in diabetic pregnant women with vascular compromise. Am J Obstet Gynecol 149: 617-623

15. Grenfell A, Brudenell JM, Doddridge MC, Watkins PJ (1986) Pregnancy in diabetic women who have proteinuria. Q J Med 228: 379-386

16. Baylis C (1981) Glomerular filtration and volume regulation in gravid animal models. Clin Obstet Gynaecol 1: 798813 